TAIWANESE JOURNAL OF MATHEMATICS

Vol. 12, No. 5, pp. 1165-1176, August 2008

This paper is available online at http://www.tjm.nsysu.edu.tw/

\title{
REGULAR VECTOR-FIELDS IN BANACH SPACES
}

\author{
Simeon Reich and Alexander J. Zaslavski
}

\begin{abstract}
It is known that regular vector-fields generate convergent discrete and continuous descent methods for minimizing functions. In this note we present necessary and sufficient conditions for regularity of descent vectorfields associated with convex and Lipschitz functions defined on general $\mathrm{Ba}-$ nach spaces.
\end{abstract}

\section{Introduction and Statement of Results}

Descent methods are an important topic in optimization theory and in dynamical systems; see, for example, [1, 2, 4, 7-12]. Given a continuous convex function $f$ on a Banach space $X$, we associate with $f$ a complete metric space $\mathcal{A}$ of mappings $V: X \rightarrow X$ such that $f^{0}(x, V x) \leq 0$ for all $x \in X$. Here $f^{0}(x, u)$ is the righthand derivative of $f$ at $x$ in the direction of $u \in X$. We call such mappings descent vector-fields (with respect to $f$ ). In [10] we identified a regularity property of such vector-fields and showed that regular vector-fields generate convergent discrete descent methods. This has turned out to be true for continuous descent methods as well [12]. Such results are significant because most of the elements in $\mathcal{A}$ are, in fact, regular [10]. Here by "most" we mean an everywhere dense $G_{\delta}$ subset of $\mathcal{A}$ (cf., for example, [5, 6, 9-11, 13]). Thus it is important to know when a given descent vector-field $V: X \rightarrow X$ is regular. In this paper we establish necessary and sufficient conditions for regularity: see Theorems 1.1-1.4 below.

More precisely, let $(X,\|\cdot\|)$ be a Banach space and let $\left(X^{*},\|\cdot\|_{*}\right)$ be its dual.

For each $g: X \rightarrow R^{1}$, set $\inf (g):=\inf \{g(z): z \in X\}$.

Let $U$ be a nonempty, open subset of $X$ and let $f: U \rightarrow R^{1}$ be a locally Lipschitz function.

Received July 8, 2006, accepted November 19, 2006.

Communicated by Sen-Yen Shaw.

2000 Mathematics Subject Classification: 37L99, 54E50, 54E52, 90C25, 90C26.

Key words and phrases: Banach space, Convex function, Descent method, Lipschitz function, Minimization problem, Regular vector-field. 
For each $x \in U$, let

$$
f^{0}(x, h)=\limsup _{t \rightarrow 0^{+}, y \rightarrow x}[f(y+t h)-f(y)] / t, \quad h \in X,
$$

be the Clarke derivative of $f$ at the point $x$, and let

$$
\partial f(x)=\left\{l \in X^{*}: f^{0}(x, h) \geq l(h) \text { for all } h \in X\right\}
$$

be the Clarke subdifferential of $f$ at $x$.

For each $x \in U$, set [14]

$$
\Xi_{f}(x):=\inf \left\{f^{0}(x, u): u \in X,\|u\| \leq 1\right\} .
$$

Clearly, $\Xi_{f}(x) \leq 0$ for all $x \in X$ and $\Xi_{f}(x)=0$ if and only if $0 \in \partial f(x)$.

For each $x \in U$, set [11]

$$
\tilde{\Xi}_{f}(x):=\inf \left\{f^{0}(x, h): h \in X,\|h\|=1\right\} .
$$

Let $x \in U$. Clearly, $\tilde{\Xi}_{f}(x) \geq \Xi_{f}(x)$ and $0 \in \partial f(x)$ if and only if $\tilde{\Xi}_{f}(x) \geq 0$. In Section 2 we will prove the following two propositions.

Proposition 1.1. Let $x \in U$. If $\tilde{\Xi}_{f}(x) \geq 0$, then $\Xi_{f}(x)=0$. If $\tilde{\Xi}_{f}(x)<0$, then $\Xi_{f}(x)=\tilde{\Xi}_{f}(x)$.

Proposition 1.2. For each $x \in U$,

$$
\Xi_{f}(x)=-\inf \left\{\|l\|_{*}: l \in \partial f(x)\right\} .
$$

Assume now that $f: X \rightarrow R^{1}$ is a continuous and convex function which is bounded from below. It is known that $f$ is locally Lipschitz. It is also known [3, Ch. 2, Sec. 2] that in this case

$$
f^{0}(x, h)=\lim _{t \rightarrow 0^{+}}[f(x+t h)-f(x)] / t, \quad x, h \in X .
$$

A mapping $V: X \rightarrow X$ is called regular [10] if $V$ is bounded on every bounded subset of $X, f^{0}(x, V x) \leq 0$ for all $x \in X$, and if for any natural number $n$, there exists a positive number $\delta(n)$ such that for each $x \in X$ satisfying $\|x\| \leq n$ and $f(x) \geq \inf (f)+1 / n$, we have

$$
f^{0}(x, V x) \leq-\delta(n) .
$$

Theorem 1.1. Let $f: X \rightarrow R^{1}$ be a convex and continuous function which is bounded from below, let $\bar{x} \in X$ satisfy

$$
f(\bar{x})=\inf \{f(z): z \in X\},
$$


and let the following property hold:

(P1) for every sequence $\left\{y_{i}\right\}_{i=1}^{\infty} \subset X$ satisfying $\lim _{i \rightarrow \infty} f\left(y_{i}\right)=f(\bar{x}), \lim _{i \rightarrow \infty} y_{i}$ $=\bar{x}$ in the norm topology.

For each natural number $n$, let $\phi_{n}:[0, \infty) \rightarrow[0, \infty)$ be an increasing function such that $\phi_{n}(0)=0$ and the following property holds:

$(P 2)$ for each $\epsilon>0$, there exists $\delta:=\delta(\epsilon, n)>0$ such that for each $t \geq 0$ satisfying $\phi_{n}(t) \leq \delta$, the inequality $t \leq \epsilon$ holds.

If $V: X \rightarrow X$ is bounded on bounded subsets of $X$,

$$
f^{0}(x, V x) \leq 0 \text { for all } x \in X,
$$

and if for each natural number $n$ and each $x \in X$ satisfying $\|x\| \leq n$, we have

$$
f^{0}(x, V x) \leq-\phi_{n}\left(-\Xi_{f}(x)\right),
$$

then $V$ is regular.

Theorem 1.2. Assume that $f: X \rightarrow R^{1}$ is a convex and continuous function, $\bar{x} \in X$,

$$
f(\bar{x})=\inf (f),
$$

property $(P 1)$ holds and the following property also holds:

(P3) if $\left\{x_{i}\right\}_{i=1}^{\infty} \subset X$ converges to $\bar{x}$ in the norm topology, then $\lim _{i \rightarrow \infty} \Xi_{f}\left(x_{i}\right)$ $=0$.

Assume that $V: X \rightarrow X$ is regular and let $n \geq 1$ be an integer. Then there exists an increasing function $\phi_{n}:[0, \infty) \rightarrow[0, \infty)$ such that $\phi_{n}(0)=0$, property (P2) holds, and for each $x \in X$ satisfying $\|x\| \leq n$, we have

$$
f^{0}(x, V x) \leq-\phi_{n}\left(-\Xi_{f}(x)\right) .
$$

Assume now that $f: X \rightarrow R^{1}$ is merely locally Lipschitz. In this case a mapping $V: X \rightarrow X$ is called regular [11] if $V$ is bounded on every bounded subset of $X$,

$$
f^{0}(x, V x) \leq 0 \text { for all } x \in X,
$$

and for any natural number $n$, there exists $\delta(n)>0$ such that for each $x \in X$ satisfying $\|x\| \leq n$ and $\Xi_{f}(x) \leq-1 / n$, we have $f^{0}(x, V x) \leq-\delta(n)$.

Theorem 1.3. Let $f: X \rightarrow R^{1}$ be a locally Lipschitz function. For each natural number $n$, let $\phi_{n}:[0, \infty) \rightarrow[0, \infty)$ be an increasing function such that $\phi_{n}(0)=0$ and property $(P 2)$ holds.

Assume that $V: X \rightarrow X$ is bounded on every bounded subset of $X$,

$$
f^{0}(x, V x) \leq 0 \text { for all } x \in X
$$


and for each natural number $n$ and each $x \in X$ satisfying $\|x\| \leq n$, we have

$$
f^{0}(x, V x) \leq-\phi_{n}\left(-\Xi_{f}(x)\right) .
$$

Then $V$ is regular.

Theorem 1.4. Assume that the function $f: X \rightarrow R^{1}$ is locally Lipschitz and that $V: X \rightarrow X$ is regular.

Then for each natural number $n$, there exists an increasing function $\phi_{n}$ : $[0, \infty) \rightarrow[0, \infty)$ such that $(P 2)$ holds and for each natural number $n$ and each $x \in X$ satisfying $\|x\| \leq n$, (1.10) holds.

\section{Proofs of Propositions 1.1 AND 1.2}

Proof of Proposition 1.1. Assume that $\tilde{\Xi}_{f}(x) \geq 0$. Then $0 \in \partial f(x)$ and $\Xi_{f}(x)=0$. Assume that $\tilde{\Xi}_{f}(x)<0$. Then by definition (see (1.4)),

$$
\inf \left\{f^{0}(x, h): h \in X,\|h\|=1\right\}=\tilde{\Xi}_{f}(x)<0 .
$$

By (2.1) and the homogeneity of $f^{0}(x, \cdot)$,

$$
f^{0}(x, h) \geq \tilde{\Xi}_{f}(x)\|h\| \text { for all } h \in X .
$$

By (2.1), (1.4), (1.3) and (2.2),

$$
\begin{aligned}
0> & \tilde{\Xi}_{f}(x) \geq \Xi_{f}(x)=\inf \left\{f^{0}(x, h): h \in X,\|h\| \leq 1\right\} \\
& \geq \inf \left\{\tilde{\Xi}_{f}(x)\|h\|: h \in X,\|h\| \leq 1\right\}=\tilde{\Xi}_{f}(x) .
\end{aligned}
$$

This implies that

$$
\tilde{\Xi}_{f}(x)=\Xi_{f}(x),
$$

as claimed. Proposition 1.1 is proved.

We precede the proof of Proposition 1.2 with the following lemma.

Lemma 2.1. Let $x \in U$ and $c>0$. Then the following are equivalent:

(i) $\Xi_{f}(x) \geq-c$; (ii) $\tilde{\Xi}_{f}(x) \geq-c$; (iii) there is $l \in \partial f(x)$ such that $\|l\|_{*} \leq c$.

Proof. By Proposition 1.1,

$$
\Xi_{f}(x) \geq-c \text { if and only if } \tilde{\Xi}_{f}(x) \geq-c .
$$


We have by (1.4) that $\tilde{\Xi}_{f}(x) \geq-c$ if and only if

$$
f^{0}(x, h) \geq-c \text { for all } h \in X \text { satisfying }\|h\|=1,
$$

which is, in its turn, equivalent to the following relation:

$$
f^{0}(x, h) \geq-c\|h\| \text { for all } h \in X .
$$

Rewriting this last inequality as

$$
f^{0}(x, h)+c|| h \| \geq 0 \text { for all } h \in X,
$$

we see that it is equivalent to the inclusion

$$
0 \in \partial f(x)+c\left\{l \in X^{*}:\|l\|_{*} \leq 1\right\} .
$$

Thus we have proved that (ii) is equivalent to (iii). This completes the proof of Lemma 2.1.

Proof of Proposition 1.2. Clearly, equality (1.5) holds if either one of its sides equals zero. Therefore we need to prove (1.5) only in the case where

$$
\Xi_{f}(x)<0 \text { and } \inf \left\{\left.|l|\right|_{*}: l \in \partial f(x)\right\}>0 .
$$

Assume that (2.3) holds. By Lemma 2.1, there is $\bar{l}$ such that

$$
\bar{l} \in \partial f(x) \text { and }\|\bar{l}\|_{*} \leq-\Xi_{f}(x) .
$$

Hence

$$
-\inf \left\{\|l\|_{*}: l \in \partial f(x)\right\} \geq-\|\bar{l}\|_{*} \geq \Xi_{f}(x) .
$$

Let $\epsilon$ be any positive number. There is $l_{\epsilon} \in \partial f(x)$ such that

$$
\left\|l_{\epsilon}\right\|_{*} \leq \inf \left\{\|l\|_{*}: l \in \partial f(x)\right\}+\epsilon
$$

By (2.6) and Lemma 2.1,

$$
\Xi_{f}(x) \geq-\epsilon-\inf \left\{\|l\|_{*}: l \in \partial f(x)\right\} .
$$

Since $\epsilon$ is any positive number, we conclude that

$$
\Xi_{f}(x) \geq-\inf \left\{\|l\|_{*}: l \in \partial f(x)\right\} .
$$

Together with (2.5), this completes the proof of Proposition 1.2. 


\section{An Auxiliary Result}

Proposition 3.1. Let $g: X \rightarrow R^{1}$ be a convex continuous function, $\bar{x} \in X$,

$$
g(\bar{x})=\inf \{g(z): z \in X\},
$$

and let the following property hold:

(P4) for any sequence $\left\{y_{i}\right\}_{i=1}^{\infty} \subset X$ satisfying $\lim _{i \rightarrow \infty} g\left(y_{i}\right)=g(\bar{x})$, we have $\lim _{i \rightarrow \infty}\left\|y_{i}-\bar{x}\right\|=0$.

Assume that $\left\{x_{i}\right\}_{i=1}^{\infty} \subset X$,

$$
\sup \left\{\left\|x_{i}\right\|: i=1,2, \ldots\right\}<\infty \text { and } \lim _{i \rightarrow \infty} \Xi_{g}\left(x_{i}\right)=0 .
$$

Then $\lim _{i \rightarrow \infty}\left\|x_{i}-\bar{x}\right\|=0$.

Proof. By (3.2) and Proposition 1.2, there exists a sequence $\left\{l_{i}\right\}_{i=1}^{\infty} \subset X_{*}$ such that

$$
\lim _{i \rightarrow \infty}\left\|l_{i}\right\|_{*}=0 \text { and } l_{i} \in \partial g\left(x_{i}\right) \text { for all integers } i \geq 1 .
$$

Choose a number $M>0$ such that

$$
\left\|x_{i}\right\| \leq M \text { for all integers } i \geq 1
$$

and let $i \geq 1$ be an integer. By (3.3),

$$
g(z)-l_{i}(z) \geq g\left(x_{i}\right)-l_{i}\left(x_{i}\right) \text { for all } z \in X .
$$

It follows from (3.5), (3.4) and (3.3) that

$$
\begin{gathered}
g(\bar{x})-g\left(x_{i}\right)=g(\bar{x})-l_{i}(\bar{x})-\left(g\left(x_{i}\right)-l_{i}\left(x_{i}\right)\right)+l_{i}\left(\bar{x}-x_{i}\right) \\
\geq l_{i}\left(\bar{x}-x_{i}\right) \geq-\left\|l_{i}\right\|\left\|\bar{x}-x_{i}\right\| \geq-\| l_{i} \mid(M+\|\bar{x}\|) \rightarrow 0 \text { as } i \rightarrow \infty
\end{gathered}
$$

and therefore

$$
\liminf _{i \rightarrow \infty}\left(g(\bar{x})-g\left(x_{i}\right)\right) \geq 0 .
$$

Together with (P4) this implies that $\lim _{i \rightarrow \infty}\left\|x_{i}-\bar{x}\right\|=0$. Proposition 3.1 is proved.

\section{Proof of Theorem 1.1}

To show that $V$ is regular, let $n$ be a natural number. We have to find a positive 
number $\delta=\delta(n)$ such that for each $x \in X$ satisfying $\|x\| \leq n$ and $f(x) \geq$ $\inf (f)+1 / n$,

$$
f^{0}(x, V x) \leq-\delta
$$

Let us assume the contrary. Then for each natural number $k$, there exists $x_{k} \in X$ satisfying

$$
\left\|x_{k}\right\| \leq n, f\left(x_{k}\right) \geq \inf (f)+1 / n
$$

and

$$
f^{0}\left(x_{k}, V x_{k}\right)>-1 / k
$$

It follows from (4.2), (4.1) and (1.8) that for each natural number $k$,

$$
-k^{-1}<f^{0}\left(x_{k}, V x_{k}\right) \leq-\phi_{n}\left(-\Xi_{f}\left(x_{k}\right)\right)
$$

and hence $\phi_{n}\left(-\Xi_{f}\left(x_{k}\right)\right)<k^{-1}$.

Together with (P2) this inequality implies that $\lim _{k \rightarrow \infty} \Xi_{f}\left(x_{k}\right)=0$. When combined with Proposition 3.1 and (4.1), this convergence implies that $\lim _{k \rightarrow \infty} \| x_{k}-$ $\bar{x} \|=0$. Since $f$ is continuous,

$$
\lim _{k \rightarrow \infty} f\left(x_{k}\right)=f(\bar{x})=\inf (f)
$$

This, however, contradicts (4.1). The contradiction we have reached proves that $V$ is indeed regular, as required.

\section{Proof of Theorem 1.2}

In what follows we make the convention that the infimum over the empty set is infinity. Set $\phi_{n}(0)=0$ and let $t>0$. Put

$$
\phi_{n}(t)=\min \left\{\inf \left\{-f^{0}(x, V x): x \in X,\|x\| \leq n \text { and } \Xi_{f}(x) \leq-t\right\}, 1\right\} .
$$

Clearly, $\phi_{n}:[0, \infty) \rightarrow[0,1]$ is well-defined and increasing.

We show that for each $x \in X$ satisfying $\|x\| \leq n$,

$$
f^{0}(x, V x) \leq-\phi_{n}\left(-\Xi_{f}(x)\right) .
$$

Let $x \in X$ with $\|x\| \leq n$. If $\Xi_{f}(x)=0$, then it is obvious that (5.2) holds.

Assume now that

$$
\Xi_{f}(x)<0
$$


Then by (5.1), (5.3) and the inequality $\|x\| \leq n$,

$$
\begin{gathered}
\phi_{n}\left(-\Xi_{f}(x)\right)=\min \left\{\inf \left\{-f^{0}(y, V y): y \in X,\|y\| \leq n \text { and } \Xi_{f}(y) \leq \Xi_{f}(x)\right\}, 1\right\} \\
\leq \min \left\{1,-f^{0}(x, V x)\right\} \leq-f^{0}(x, V x)
\end{gathered}
$$

and hence

$$
f^{0}(x, V x) \leq-\phi_{n}\left(-\Xi_{f}(x)\right) .
$$

Thus (5.2) holds for each $x \in X$ satisfying $\|x\| \leq n$.

Next we show that (P2) holds. To this end, let $\epsilon>0$. We will show that there is $\delta>0$ such that for each $t \geq 0$ satisfying $\phi_{n}(t) \leq \delta$, the inequality $t \leq \epsilon$ holds.

Assume the contrary. Then for each natural number $i$, there exists $t_{i} \geq 0$ such that

$$
\phi_{n}\left(t_{i}\right) \leq(4 i)^{-1}, t_{i}>\epsilon
$$

By (5.4) and (5.1), for each natural number $i$, there exists a point $x_{i} \in X$ such that

$$
\left\|x_{i}\right\| \leq n, \Xi_{f}\left(x_{i}\right) \leq-t_{i}<-\epsilon,
$$

and

$$
f^{0}\left(x_{i}, V x_{i}\right) \geq-(2 i)^{-1}
$$

Now it follows from (5.5), (5.6) and the definition of regularity that

$$
\lim _{i \rightarrow \infty} f\left(x_{i}\right)=f(\bar{x})
$$

Together with (P1) this implies that $\lim _{i \rightarrow \infty}\left\|x_{i}-\bar{x}\right\|=0$. When combined with (P3), this inequality implies that $\lim _{i \rightarrow \infty} \Xi_{f}\left(x_{i}\right)=0$. This, however, contradicts (5.5). The contradiction we have reached proves Theorem 1.2.

\section{Proof of Theorem 1.3}

Let $n$ be a natural number. We need to show that there exists $\delta>0$ such that for each $x \in X$ satisfying

$$
\|x\| \leq n \text { and } \Xi_{f}(x)<-1 / n,
$$

we have

$$
f^{0}(x, V x) \leq-\delta .
$$


Let us assume the contrary. Then for each natural number $k$, there exists $x_{k} \in X$ such that

$$
\left\|x_{k}\right\| \leq n, \Xi_{f}\left(x_{k}\right) \leq-1 / n
$$

and

$$
f^{0}\left(x_{k}, V x_{k}\right)>-1 / k .
$$

By (6.2) and (1.10),

$$
-1 / k<f^{0}\left(x_{k}, V x_{k}\right) \leq-\phi_{n}\left(-\Xi_{f}\left(x_{k}\right)\right)
$$

and

$$
\phi_{n}\left(-\Xi_{f}\left(x_{k}\right)\right) \leq 1 / k .
$$

It now follows from (6.3) and property (P2) that

$$
\limsup _{k \rightarrow \infty}\left(-\Xi_{f}\left(x_{k}\right)\right)=0
$$

and

$$
\lim _{k \rightarrow \infty} \Xi_{f}\left(x_{k}\right)=0 .
$$

The last equality contradicts (6.2) and this contradiction proves Theorem 1.3.

\section{Proof of Theorem 1.4}

Set $\phi_{n}(0)=0$ and let $t>0$. Define

$$
\phi_{n}(t)=\min \left\{\inf \left\{-f^{0}(x, V x): x \in X,\|x\| \leq n, \Xi_{f}(x) \leq-t\right\}, 1\right\} .
$$

Clearly, $\phi:[0, \infty) \rightarrow[0,1]$ is well-defined and increasing.

We show that for each $x \in X$ satisfying $\|x\| \leq n$,

$$
f^{0}(x, V x) \leq-\phi_{n}\left(-\Xi_{f}(x)\right) .
$$

Let $x \in X$ with

$$
\|x\| \leq n
$$

If $\Xi_{f}(x)=0$, then (7.2) clearly holds. Assume now that

$$
\Xi_{f}(x)<0 .
$$


Then by (7.1), (7.2), (7.3) and (7.4),

$$
\begin{aligned}
& \phi_{n}\left(-\Xi_{f}(x)\right)=\min \left\{\inf \left\{-f^{0}(y, V y): y \in X,\|y\| \leq n, \Xi_{f}(y) \leq \Xi_{f}(x)\right\}, 1\right\} \\
& \leq \min \left\{1,-f^{0}(x, V x)\right\} \leq-f^{0}(x, V x)
\end{aligned}
$$

and hence (7.2) holds for all $x \in X$ satisfying $\|x\| \leq n$, as claimed.

Now we show that property (P2) also holds. To this end, let $\epsilon$ be positive.

We claim that there is $\delta>0$ such that for each $t \geq 0$ satisfying $\phi_{n}(t) \leq \delta$, the inequality $t \leq \epsilon$ holds.

Let us assume the contrary. Then for each natural number $i$, there exists $t_{i} \geq 0$ such that

$$
\phi\left(t_{i}\right) \leq(4 i)^{-1}, t_{i}>\epsilon .
$$

Let $i$ be a natural number. By (7.5) and (7.1), there exists $x_{i} \in X$ such that

$$
\left\|x_{i}\right\| \leq n, \Xi_{f}\left(x_{i}\right) \leq-t_{i}<-\epsilon,
$$

and

$$
-f^{0}\left(x_{i}, V x_{i}\right) \leq(2 i)^{-1}
$$

Clearly,

$$
f^{0}\left(x_{i}, V x_{i}\right) \geq-(2 i)^{-1} .
$$

Choose a natural number $p$ such that

$$
p>n \text { and } 1 / p<\epsilon .
$$

Since $V$ is regular, there is $\delta>0$ such that

$$
\text { if } x \in X,\|x\| \leq p \text { and } \Xi_{f}(x)<-1 / p \text {, then } f^{0}(x, V x)<-\delta \text {. }
$$

Choose a natural number $j$ such that

$$
1 / j<\delta
$$

Then for all integers $i \geq j$, it follows from (7.6) and (7.8) that

$$
\Xi_{f}\left(x_{i}\right)<-\epsilon<-1 / p \text { and }\left\|x_{i}\right\| \leq p .
$$

Together with (7.9) and (7.10), this implies that for all integers $i \geq j$,

$$
f^{0}\left(x_{i}, V x_{i}\right)<-\delta<-j^{-1}<-(i)^{-1} .
$$

Since this contradicts (7.7), the proof of Theorem 1.4 is complete. 


\section{ACKNOWLEDGMENT}

The research of the first author was partially supported by the Fund for the Promotion of Research at the Technion and by the Technion VPR Fund - B. and G. Greenberg Research Fund (Ottawa).

\section{REFERENCES}

1. S. Aizicovici, S. Reich and A. J. Zaslavski, Convergence theorems for continuous descent methods, J. Evol. Equ., 4 (2004), 139-156.

2. S. Aizicovici, S. Reich and A. J. Zaslavski, Most continuous descent methods converge, Arch. Math. (Basel), 85 (2005), 268-277.

3. F. H. Clarke, Optimization and Nonsmooth Analysis, SIAM, Philadelphia, 1990.

4. H. B. Curry, The method of steepest descent for nonlinear minimization problems, Quart. Appl. Math., 2 (1944), 258-261.

5. F. S. De Blasi and J. Myjak, Sur la convergence des approximations successives pour les contractions non linéaires dans un espace de Banach, C. R. Acad. Sci. Paris, 283 (1976), 185-187.

6. F. S. De Blasi and J. Myjak, Generic flows generated by continuous vector fields in Banach spaces Adv. Math., 50 (1983), 266-280.

7. J.-B. Hiriart-Urruty and C. Lemaréchal, Convex Analysis and Minimization Algorithms, Springer, Berlin, 1993.

8. J. W. Neuberger Sobolev Gradients and Differential Equations, Lecture Notes in Math., 1670, Springer, Berlin, 1997.

9. S. Reich and A. J. Zaslavski, Generic convergence of descent methods in Banach spaces Math. Oper. Research, 25 (2000), 231-242.

10. S. Reich and A. J. Zaslavski, The set of divergent descent methods in a Banach space is $\sigma$-porous, SIAM J. Optim., 11 (2001), 1003-1018.

11. S. Reich and A. J. Zaslavski, Porosity of the set of divergent descent methods Nonlinear Anal., 47 (2001), 3247-3258.

12. S. Reich and A. J. Zaslavski, Two convergence results for continuous descent methods Electron. J. Differential Equations, 2003 (2003), 1-11.

13. G. Vidossich, Most of the successive approximations do converge, J. Math. Anal. Appl., 45 (1974), 127-131.

14. A. J. Zaslavski, On critical points of Lipschitz functions on smooth manifolds, Siberian Math. J., 22 (1981), 63-68. 
Simeon Reich and Alexander J. Zaslavski

Department of Mathematics,

The Technion-Israel Institute of Technology,

32000 Haifa, Israel

E-mail: sreich@tx.technion.ac.il ajzasl@tx.technion.ac.il 